\title{
INFLUENCE OF THE MOMENTUM COMPACTION UPON LANDAU CAVITY STABILITY*
}

\author{
R. A. Bosch, ${ }^{\#}$ K. J. Kleman and J. J. Bisognano, \\ Synchrotron Radiation Center, University of Wisconsin-Madison, \\ 3731 Schneider Dr., Stoughton, WI 53589, USA
}

\section{Abstract}

A fourth-harmonic "Landau" radiofrequency (RF) cavity suppresses coupled bunch instabilities and increases the beam lifetime of the Aladdin electron storage ring. When the storage ring is operated with a small momentum compaction, instabilities limit the utility of the Landau cavity. Analytic modeling and simulations suggest that instabilities result from fast mode-coupling between the dipole and quadrupole Robinson modes.

\section{INTRODUCTION}

An RF cavity with resonant frequency near a harmonic of the fundamental RF cavity may increase Landau damping of synchrotron oscillations and lengthen the bunch, thereby suppressing coupled bunch instabilities and increasing the Touschek lifetime [1-3]. A Landau cavity may be operated in passive mode, where its voltage is induced by the beam current, or active mode, where its voltage is maintained by an RF power supply and feedback. Quiet operation requires the avoidance of Robinson instabilities, which are coupled bunch instabilities where all bunches oscillate in unison.

Since 1995, a fourth-harmonic cavity has been operated at the Aladdin $300 \mathrm{~mA}, 800 \mathrm{MeV}$ electron storage ring, suppressing coupled-bunch instabilities and doubling the beam lifetime [3]. When using a low-emittance lattice in which the momentum compaction $\alpha$ is decreased by a factor of $\sim 8$, Landau-cavity operation is limited by apparent Robinson instabilities. Here, we study Robinson instabilities using analytic modeling and simulations. The modeling suggests that the small momentum compaction increases coupling between the dipole and quadrupole Robinson modes, resulting in fast modecoupling instabilities.

\section{ROBINSON INSTABILITIES}

The natural frequency of dipole collective oscillations in the synchrotron potential formed by two RF cavities may be estimated by approximating the dipole motion as rigid [1]. We model Robinson instabilities by substituting this frequency in place of the linear synchrotron frequency in formulas appropriate for a harmonic synchrotron

\footnotetext{
* Work supported by NSF grant DMR-0084402

\# Email: bosch@src.wisc.edu
}

potential. Coupling between the dipole and quadrupole modes is included in the model; the coupled modes are referred to as coupled-dipole and coupled-quadrupole modes. A fast mode-coupling instability is predicted when the frequencies of the two modes converge.

We consider the case where external power is supplied to RF cavities by operating them in the "compensated condition" with the RF generator current in phase with the cavity voltage. Fast RF feedback is included in the equivalent RF-coupling coefficients of the fundamental and Landau cavities. In addition to analytic predictions of instability frequencies and growth rates, simulations are performed to evaluate stability. When the energy spread of the electrons exceeds its natural value by more than $10 \%$ after tracking 500,000 turns of longitudinal motion, a simulation is considered to display instability.

The analytic model is expected to predict instability frequencies and growth rates within $\sim 10 \%$, provided that the Landau-cavity voltage is sufficiently low that the longitudinal bunch profile has a single maximum, i.e. for "single-hump" bunches. For single-hump bunches, the model is in approximate agreement with simulations and experiment. For active Landau-cavity operation with double-hump bunches, simulations may display instability that is not predicted by the analytic model [1].

For an "optimally lengthened" bunch (whose length is the maximum obtainable with a single-hump bunch shape), the natural frequency of a collective oscillation is proportional to $\alpha^{3 / 4}$. With a finite beam current, the frequency of a Robinson oscillation is shifted from this value by RF fields induced by the bunch motion. This frequency shift is approximately proportional to $\alpha^{1 / 4}$. When $\alpha$ is reduced, the frequency shift may become comparable to the collective oscillation frequency, giving rise to strong coupling between the dipole and quadrupole Robinson modes [1].

\section{STANDARD ALADDIN OPERATION}

We first consider operation with a $90-\mathrm{kV}$ fundamental $\mathrm{RF}$ voltage in the standard Aladdin lattice, whose momentum compaction is 0.0335 . Aladdin parameters are given in Ref. [1]. For passive operation of the Landau cavity, analytic instability predictions are shown in Fig. 1(a) for the coupled-dipole and coupled-quadrupole Robinson modes. Simulation results shown in Fig. 1(b) agree with the analytic modeling. For currents $\geq 120 \mathrm{~mA}$, 


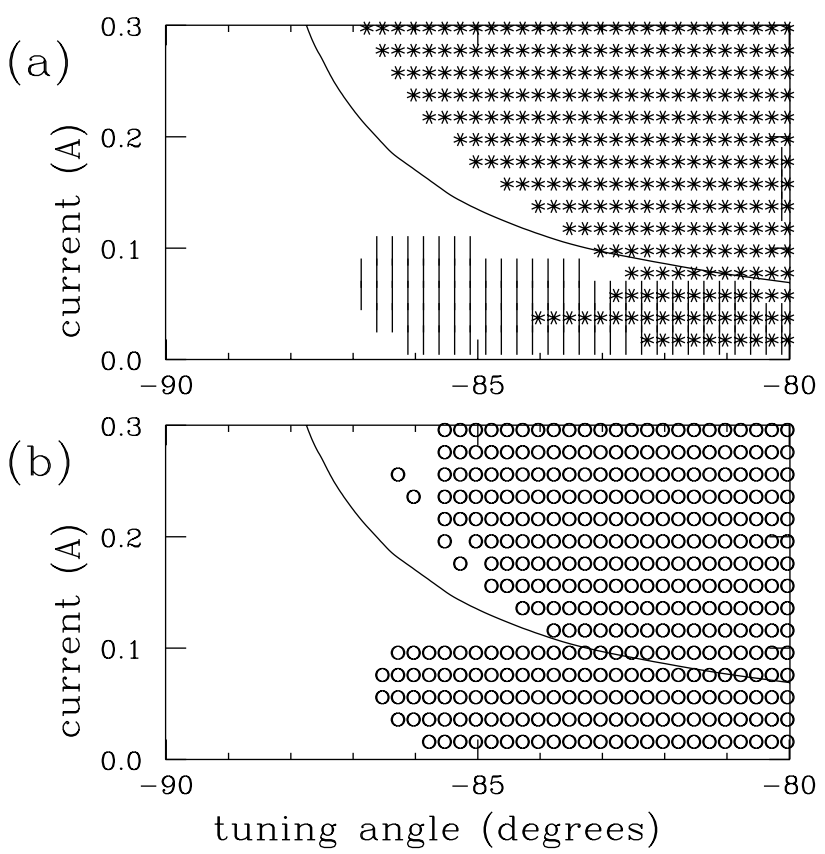

Figure 1 (a) Robinson instability predictions for passive Landau-cavity operation with the standard Aladdin lattice. A solid curve shows the parameters for optimal bunch lengthening. The dipole and quadrupole modes are perturbed by coupling, but a fast mode-coupling instability is not predicted. |: coupled-dipole instability; * : coupled-quadrupole instability. (b) Instabilities observed in 500,000-turn simulations.

stable operation is predicted with optimally lengthened bunches, in agreement with experiment [3].

For active operation of the Landau cavity with optimally lengthened bunches, analytic modeling and simulations are shown in Fig. 2 for various values of the Landau-cavity feedback, which is parameterised by the equivalent RF coupling coefficient, $\beta_{2}$. For $\beta_{2}>60$, stable operation is indicated.

The RF coupling and feedback of our Landau cavity is estimated to be equivalent to $\beta_{2}$ equalling $\sim 160$. For $\beta_{2}=$ 160 and ring currents of $0-300 \mathrm{~mA}$, analytic modeling and simulations were performed for various values of the dimensionless parameter $\xi$, which is the ratio of the Landau-cavity voltage to that which would produce an optimally lengthened bunch [2]. A coupled-bunch instability with longitudinal mode number of 1 may be excited by the Landau cavity for $\xi<0.3$; this is predicted analytically and observed in simulations [1]. For $0<\xi \leq$ 2 , Robinson instabilities are not predicted or observed in simulations. Experimentally, stable active operation with $0.5 \leq \xi \leq 1$ is obtained daily.

\section{THE LF20 LATTICE}

To lower its emittance, the storage ring may be operated using the LF20 lattice [4], whose momentum compaction of 0.0043 is a factor of $\sim 8$ smaller than that of
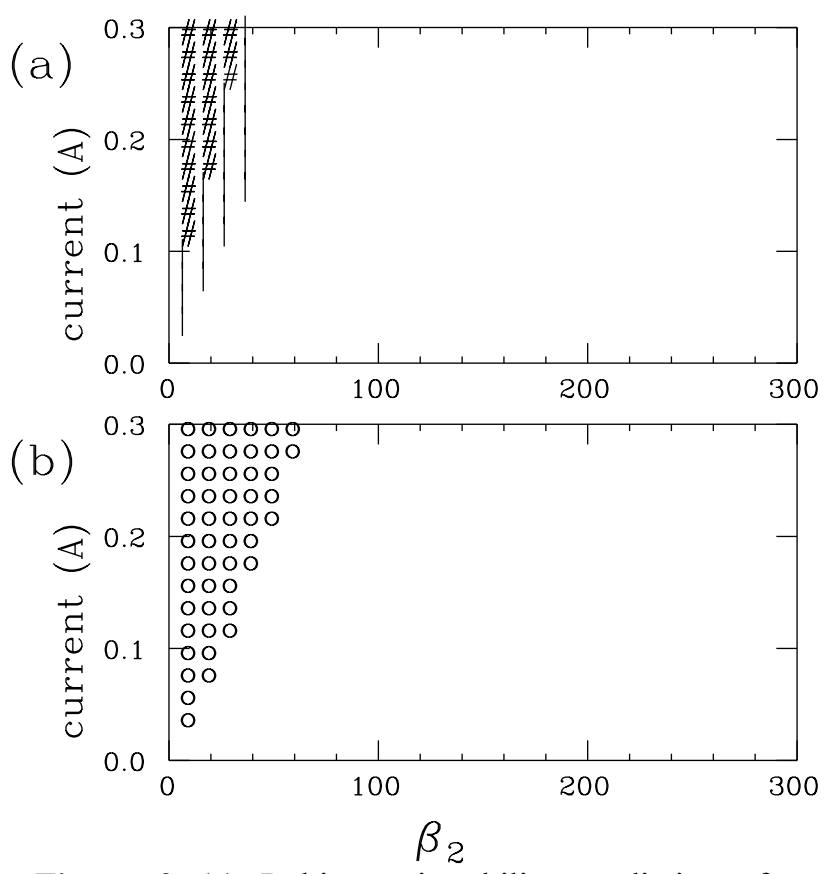

Figure 2 (a) Robinson instability predictions for active Landau-cavity operation with optimally lengthened bunches in the Aladdin lattice. | : coupleddipole instability; \# : fast mode-coupling instability.

(b) Instabilities observed in 500,000-turn simulations.

the standard Aladdin lattice; the longitudinal damping time is also reduced trivially from 13.8 to $13.5 \mathrm{~ms}$. In contrast to studies in Ref. [1], we consider LF20 operation with a $90-\mathrm{kV}$ fundamental RF voltage, identical to that of standard Aladdin operation.

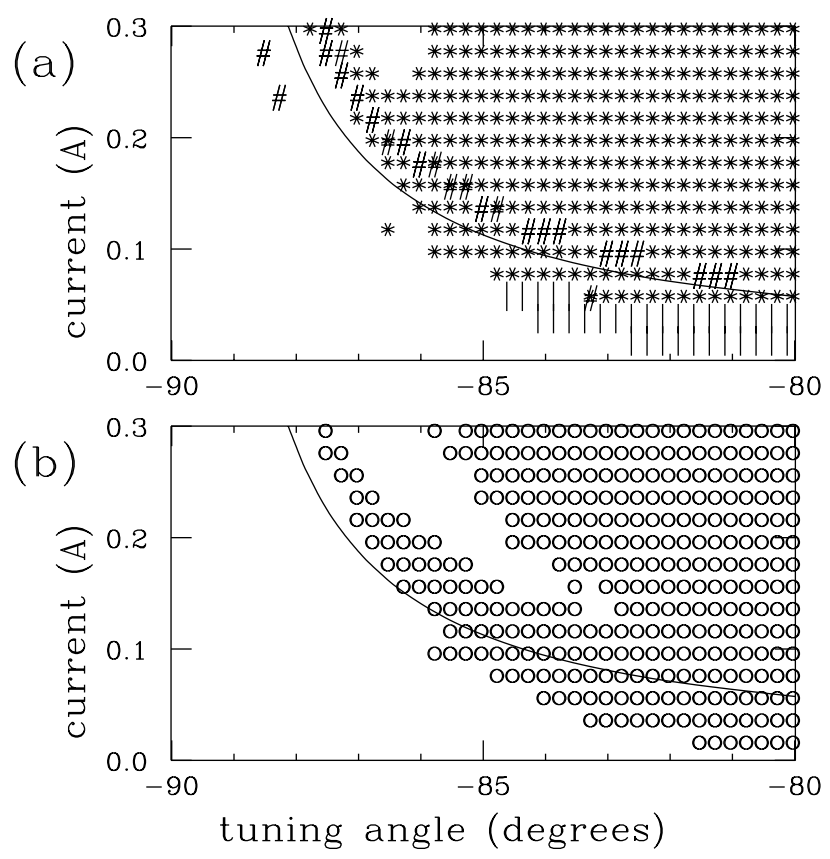

Figure 3 (a) Robinson instability predictions for passive Landau-cavity operation with the LF20 lattice. |: coupled-dipole instability; ${ }^{*}$ : coupled-quadrupole instability; \# : fast mode-coupling instability. (b) Instabilities observed in 500,000-turn simulations. 

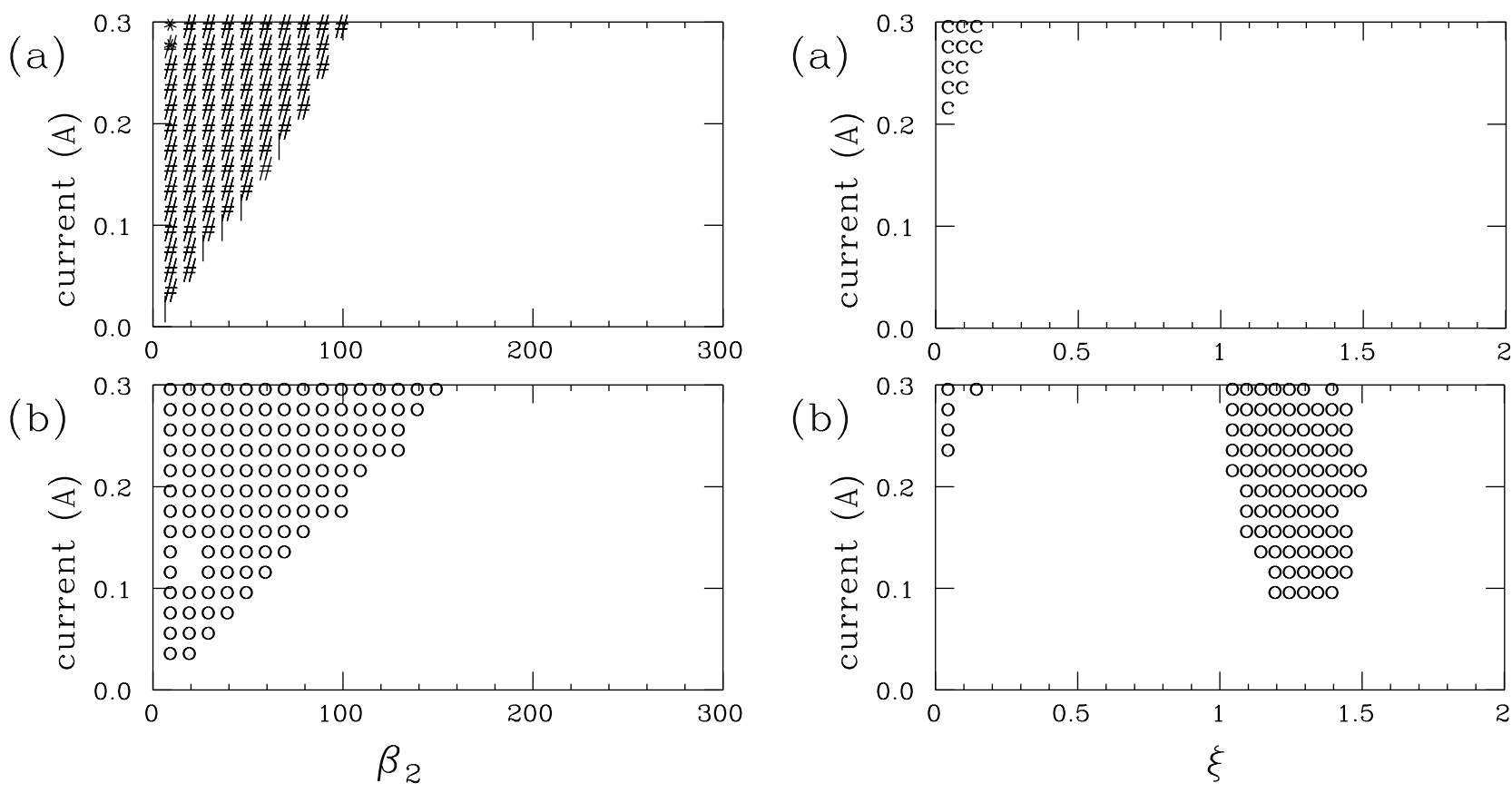

Figure 4 (a) Robinson instability predictions for active Landau-cavity operation with optimally lengthened bunches in the LF20 lattice. |: coupleddipole instability; *: coupled-quadrupole instability; \#: fast mode-coupling instability. (b) Instabilities observed in 500,000-turn simulations.

Analytic modeling of passive operation is shown in Fig. 3(a). A fast mode-coupling instability is predicted for bunches lengthened slightly beyond optimal; further lengthening is predicted to yield stable double-hump bunches for currents exceeding $240 \mathrm{~mA}$. The analytic modeling is in approximate agreement with simulations shown in Fig. 3(b). In simulations, stable double-hump bunches are obtained for currents exceeding $120 \mathrm{~mA}$, in excellent agreement with experiment.

Instability modeling for active operation with optimally lengthened bunches is shown in Fig. 4. Stable operation is indicated for currents of $0-300 \mathrm{~mA}$ when $\beta_{2} \geq 160$.

For $\beta_{2}=160$, analytic modeling and simulations are shown in Fig. 5 for $0<\xi<2$. For single-hump bunches $(\xi \leq 1)$, simulations agree with analytic modeling, while for double-hump bunches $(\xi>1)$, instabilities are more prevalent in simulations [1]. In experiments, optimally lengthened bunches are unstable when the current exceeds $\sim 100 \mathrm{~mA}$, in agreement with analytic modeling and simulations performed for $\beta_{2}$ equalling 40-80. This suggests that, in practice, the RF coupling and feedback are equivalent to $\beta_{2}<160$, insufficient to prevent modecoupling Robinson instability. A larger feedback gain may yield stable operation with optimally lengthened bunches throughout the current range of 0-300 mA.

For both lattices, analytic modeling predicts that sufficient Landau damping is obtained with optimally lengthened bunches to suppress parasitic coupled bunch

Figure 5 (a) Instability predictions for active Landau-cavity operation with the LF20 lattice and $\beta_{2}=160$. c: coupled bunch instability with longitudinal mode number of 1 . (b) Instabilities observed in 500,000 turn simulations.

instabilities from typical higher-order modes of the RF cavities. This prediction is confirmed by experiment [1].

\section{SUMMARY}

We have studied Robinson instabilities when a Landau cavity is utilized with the standard Aladdin lattice and a lattice with a smaller momentum compaction. Analytic modeling, simulations and experiments are in approximate agreement for single-hump bunch shapes, and for passive Landau cavity operation with double-hump bunches. With a small momentum compaction, increased instability results from coupling of the dipole and quadrupole Robinson modes. In analytic modeling and simulations, the stability of active Landau cavity operation with a low momentum compaction is improved by increasing the equivalent RF coupling coefficient of the Landau cavity.

\section{REFERENCES}

[1] R. A. Bosch, K. J. Kleman and J. J. Bisognano, Phys. Rev. ST Accel. Beams (to be published); and references therein.

[2] Tai-Sen F. Wang, in Proc. 1993 Particle Accelerator Conference (IEEE, Piscataway, NJ, 1993), p. 3500.

[3] K. J. Kleman, in Proc. 1995 Particle Accelerator Conference (IEEE, Piscataway, NJ, 1996), p. 1785.

[4] J. J. Bisognano, R. A. Bosch, D.E. Eisert, M.A. Green, K. J. Kleman and W. S. Trzeciak, these proceedings. 